\title{
Percepciones sobre la escuela democrática en Argentina y España'
}

\author{
Roser Grau Vidal ${ }^{2}$ \\ https://orcid.org/0000-0003-0622-1245 \\ Laura García Raga² \\ https://orcid.org/0000-0002-7242-6620 \\ Ramón López Martín² \\ http://orcid.org/0000-0001-9450-8910
}

\section{Resumen}

El estudio de las múltiples relaciones entre la educación y el sentido conceptual de la democracia sigue siendo una temática de interés. El objetivo principal del trabajo que se presenta es poner en valor el concepto, los pilares y el proceso de construcción de una escuela democrática, categorías sobre cuyos cimientos se asienta el carácter democrático de la institución escolar: la exigencia de la participación y la mejora de la convivencia. Este estudio se ha desarrollado a través de la voz de especialistas de educación de Salta (Argentina) y Valencia (España), comparando las acepciones más destacadas entre ambos países. A nivel metodológico, se ha llevado a cabo una investigación de carácter cualitativo, a través de entrevistas, encuestas y grupos focales, participando un total de 680 sujetos. Los resultados obtenidos evidencian que, pese a las diferencias detectadas, las exigencias y las necesidades de ambos países confluyen más de lo previsto. Desde ambas perspectivas se hace latente la necesidad de construir una escuela más inclusiva, participativa y formadora, a través de la mejora de la coordinación y la implicación de profesionales, familias y comunidad. Se concluye, finalmente, que una escuela democrática es una comunidad de aprendizaje donde la participación de todos (educar en democracia), aspira a convertirse en un taller práctico de convivencia (educar para la democracia).

\section{Palabras clave}

Valores democráticos - Participación - Educación para la ciudadanía - Relaciones interpersonales - Escuela democrática.

1- Esta investigación se desarrolla en el marco del programa VALi+d de Ayudas para la contratación de personal investigador en formación de carácter predoctoral, Conselleria de Educación, Cultura y Deporte, Comunitat Valenciana [2013/8197] y de forma complementaria de acuerdo con la ORDEN 64/2014, de 31 de julio, de la Consellería de Educación, Cultura y Deporte [2014/7448], ANEXO III, Becas para estancias de contratados predoctorales en centros de investigación fuera de la Comunitat Valenciana.

2- Unversitat de València, Valencia, España.Contactos: roser.grau@uv.es, laura.garcia@uv.es, rlopez@uv.es.

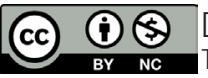

DOl: http://dx.doi.org/10.1590/S1678-4634201945188681 


\section{Insights into democratic school in Argentina and Spain}

\section{Abstract}

The study of the multiple links between education and the conceptual meaning of democracy remains a topic of interest. This paper aims to bring to the fore the concept, the pillars and the building process of a democratic school. The foundation of these categories is where the democratic character of the educational establishment lies: the imperative for participation and the improvement of living together. This study is based on the insights of 680 education specialists from Salta (Argentina) and Valencia (Spain) and compares the most salient meanings between both countries. At the methodological level, we have carried out a qualitative research by conducting interviews, surveys and focus groups. Our findings reveal that, despite the differences detected, the demands and needs of both countries converge more often than anticipated. From both perspectives, it is clear that there is a need to build a more inclusive, participatory and formative school, by improving the coordination and involvement of professionals, families and the community. Finally, it follows that a democratic school is a learning community where everyone's participation (educating about democracy) aspires to become a hands-on workshop on living together (educating for democracy).

\section{Keywords}

Democratic values - Participation - Education for citizenship - Interpersonal relationships - Democratic school.

\section{Introducción}

En la actualidad, consolidada la situación jurídica que asegura y propicia la democracia escolar, tanto en la programación general de la educación, como en el gobierno y vida cotidiana de las instituciones docentes, debemos asumir el reto de desmenuzar y perfilar el dinámico entramado relacional en el que convergen educación y democracia; en palabras de Gimeno Sacristán (1998, p. 19), “[...] se necesitan, la una a la otra, para construirse recíprocamente”. La educación alimenta las raíces de la democracia para vivificarla y hacerla más plena, de calidad, luchando contra la anomia social y la falta de compromiso ciudadano. Por otro lado, la democracia proporciona un escenario de referencia adecuado para que los procesos formativos desarrollen los valores humanos y conviertan al individuo en un ser social, bajo los principios fundamentales de libertad, igualdad, justicia social y pluralismo cultural. Esta directriz pedagógica, en el contexto actual de progresivo incremento cuantitativo de los niveles de escolarización básica, hasta alcanzar los niveles máximos en buena parte de los países iberoamericanos, exige -ahora- políticas de calidad educativa orientadas a la consecución real de los principios anunciados. 
Desde esta perspectiva, el principal objetivo del trabajo se centra en realizar una aproximación reflexiva sobre las etiquetas o componentes conformadores de las dos categorias fundamentales de una escuela democrática: participación y convivencia, tratando de dibujar los cimientos sobre los que ésta se construye y concretar los valores competenciales adecuados para dicha construcción. A la revisión de la literatura científica de la temática en cuestión, se añade el análisis de los resultados de un estudio de campo realizado en Salta (Argentina) y Valencia (España), que pone de manifiesto las percepciones de especialistas de la educación (profesorado de distintos niveles educativos y estudiantes de titulaciones universitarias relacionadas con la educación) sobre el concepto, las bases y los aspectos necesarios para el desarrollo de una escuela democrática.

A lo largo de los siguientes apartados se reflexiona acerca de los fundamentos que resultan necesarios para comprender e impulsar la construcción de una escuela democrática: la exigencia de la participación y la mejora de la convivencia, en la doble vertiente relacional de una educación en y para la democracia, es decir, la intervención comprometida de todos los sectores involucrados en la gestión académica e institucional de los centros docentes, y -como el reverso de una misma moneda- el uso de esos espacios y tiempos como un taller práctico de aprendizaje cívico orientado al ejercicio futuro de una ciudadanía comprometida y responsable.

\section{La apuesta por una ciudadanía participativa}

El concepto de democracia implica el máximo posible de participación de la ciudadanía en las decisiones públicas, asumiendo el poder de reflexionar, debatir y, en numerosas ocasiones, decidir. Por ello, si analizamos el concepto desde una perspectiva pedagógica, es responsabilidad del sistema educativo preparar a la ciudadanía para ejercer la democracia; la educación, como recoge el ordenamiento constitucional español, debe ser un instrumento al servicio de la convivencia democrática y la escuela, como primer escenario formativo, el espacio idóneo para el aprendizaje de las competencias necesarias.

En esta línea, Bolívar (2008) considera que una educación para la ciudadanía democrática activa es fundamental para construir una sociedad libre, tolerante y justa, promoviendo y educando desde la escuela en valores y principios de libertad, pluralismo y derechos humanos, construyendo así un mundo más solidario y menos ignorante. Por todo ello, resulta imprescindible que desde la escuela se eduque a la ciudadanía para que aprenda a vivir en el complejo mundo actual, con el propósito de participar en una sociedad cambiante, así como apostar por gestionar y defender democráticamente un sistema de valores en el que está integrada la tolerancia, la justicia y el respeto a las diferencias (MAYOR ZARAGOZA, 2003).

Para abordar esta cuestión y aceptando el vínculo claro de democracia y educación, es obvio que el papel de la escuela es fundamental a la hora de aprender a vivir en una sociedad democrática. Vivir la democracia en la escuela supone experimentar en primera persona los valores democráticos, trasmitiendo a través de la educación y de la cotidianidad las actitudes de tolerancia, respeto, igualdad, solidaridad, cooperación y participación en las aulas. En esta línea, siguiendo el discurso de Apple y Beane (1997, p. 44), “[...] las escuelas democráticas tienen que estar basadas en una definición amplia 
del "nosotros", un compromiso para construir una comunidad que sea tanto de la escuela como de la sociedad en la que la escuela existe".

Se puede afirmar que “[...] sin la colaboración de la escuela, la convivencia plural y los valores sociales de la democracia, no podrán consolidarse. Sin educación para la democracia, en defınitiva, no habrá democracia" (SACRISTÁN; MURGA, 1994, p. 99). La escuela es un espacio fundamental en el que se debe ejercer la democracia, convirtiéndose en una micro-sociedad en la que la participación y la toma de decisiones pasan a ocupar el primer lugar en la escala de valores; se trata, en definitiva, de revitalizar la escuela como esfera pública (CANTARERO; MARTÍNEZ BONAFÉ; MARTÍNEZ BONAFÉ, 1999). En palabras de Tonucci (2009, p. 24) la escuela debe ser un espacio "[...] donde los niños sean reconocidos como ciudadanos y, por tanto, capaces de vivir experiencias de democracia y de ciudadanía”, por lo que hay que abrir la escuela a la participación, hacer que niños y niñas tomen decisiones, afronten las dificultades, devolviéndoles la voz y la palabra para que sean ellos y ellas responsables de parte de la gestión del centro.

En este sentido, es cierto que si una sociedad apuesta firmemente por ser democrática requiere entonces de la participación activa y crítica de todas las personas que la conforman. La escuela debe preparar esa participación ciudadana sentando las bases teóricas y ofreciendo instrumentos prácticos que permitan la experimentación en el espacio y tiempo escolar (GARCÍA PÉREZ, 2009).

Cuando se habla de participación eficaz y de cultura de participación, con el propósito de potenciarla, es necesario re-pensarla y reflexionar acerca de las ventajas de vivir en una sociedad que apuesta firmemente por la participación plena y activa. Como destaca Santos Guerra (2003), si algo se considera propio, se defiende, se respeta y se ama. De esta manera, cuando se acuerdan una serie de normas en comunidad, las cuales se han debatido de forma democrática, participando todos y todas en la toma decisiones $\mathrm{y}$ en los acuerdos, se acatan porque se considera que son necesarias, valorándolas y considerándolas adecuadas para la convivencia. Como nos señala el citado autor "[...] ante la imposición, es fácil responder con la indiferencia o con la rebeldía” (2003, p. 108).

La educación, en definitiva, no puede renunciar a la construcción de una ciudadanía activa, crítica y responsable, en el convencimiento que este tipo de ciudadanía no es algo inherente a la persona, ni puede improvisarse, sino que se aprende. Apostar por la educación supone, en su sentido más esencial, trabajar por construir ciudadanía, es decir, dotar a la totalidad de la población de las herramientas necesarias para el ejercicio activo de los derechos y la asunción de responsabilidades (LÓPEZ MARTÍN, 2013). No hay ciudadanía participativa sin educación y ésta no puede plantarse en otro referente más genérico que no sea la construcción de aquella.

\section{El aprendizaje de la convivencia}

La convivencia, el aprendizaje del vivir juntos, se ha convertido en uno de los desafíos de futuro de primer nivel para las sociedades del siglo XXI (GARCÍA RAGA; LÓPEZ MARTÍN, 2014); se trata de un objetivo que debe estar presente en todo el proceso educativo y el centro escolar, como escenario idóneo para su aprendizaje, deberá 
trabajar en la construcción de entornos pedagógicos que faciliten su adquisición. Una escuela democrática no puede entenderse sin una apelación constante a la mejora de la convivencia y al aprendizaje y práctica de los valores cívicos y hábitos democráticos. Jares (2006) señala que, para poder convivir, resulta imprescindible construir una escuela desde los presupuestos de una ciudadanía democrática, respetuosa y solidaria, con la finalidad de apostar firmemente por una cultura de paz y de no violencia, persiguiendo desde la práctica educativa los principios democráticos de la convivencia. Godás, Santos Rego y Lorenzo (2008), por otro lado, profundizan en esta idea, al exponer la relación directa que existe entre el concepto de convivencia y la calidad de las relaciones interpersonales, lo que exige conformar un adecuado clima escolar.

Resulta, por tanto, imprescindible reflexionar acerca de los pilares fundamentales que requiere la construcción de una convivencia democrática a través de la práctica educativa. Aprender a convivir implica, en primer lugar, apostar por los derechos humanos, aceptando a los demás como seres dignos e iguales (TOURIÑÁN, 2010; SILVA; TAVARES, 2011); impulsar los valores de respeto mutuo y la capacidad de sacrificio, por uno mismo y por las personas que le rodean. Por ello, la tolerancia, la responsabilidad, la solidaridad, la libertad, la equidad, el compromiso y el diálogo, la justicia o el desarrollo de la cooperación, entre otros, como valores necesarios para vivir en sociedad, deberán ser transmitidos y practicados por las instituciones educativas (VIGUER; SOLÈ, 2012), en el compromiso de garantizar la construcción de una sociedad vertebrada y cohesionada.

Así pues, nos parece esencial apostar por un concepto de convivencia conformado por el entramado de los valores mencionados; una convivencia alejada de prejuicios y de estereotipos que pongan en duda los valores democráticos y fomente el respeto a la diferencia (GONZÁLEZ BAREA, 2008), evitando que ésta acabe abrazando la desigualdad; una convivencia donde se favorezca una buena comunicación (FERNÁNDEZ, 2008; REKALDE; VIZCARRA; MACAZAGA, 2014), se practique la empatía y la escucha activa para comprender a los demás; una convivencia que profundice en la cultura del diálogo como eje potenciador de un adecuado clima escolar (TIRADO; CONDE, 2015), donde el conflicto, lejos de ser algo pernicioso y antinatural, se convierta en un recurso educativo y, junto a la mediación (GARCÍA RAGA; MARTÍNEZ USARRALDE; SAHUQUILLO, 2012) y las técnicas de gestión pacífica de conflictos (TORRECILLA; OLMOS; RODRÍGUEZ, 2015), uno de los valores competenciales de una escuela democrática.

Y para todo ello, debemos remarcarlo una vez más, no podemos sustraernos a que la institución escolar, con el liderazgo de gestores y directores educativos, construya órganos de participación y estrategias que primen el intercambio y contacto con los demás; se trata de ser capaces de dinamizar la comunidad educativa para trabajar de forma conjunta, coordinando medios y personas, pero, sobre todo, participando y tomando decisiones colectivas, haciendo de la participación una realidad inexcusable (FLECHA, 2006). Es pues, en definitiva, este esfuerzo compartido orientado a la búsqueda de estrategias, actividades, prácticas y herramientas que mejoren la comunicación, propicien la participación comprometida, faciliten el diálogo responsable y alienten el respeto mutuo, la única receta para desarrollar una convivencia plena, como rasgo identitario de una escuela verdaderamente democrática. 


\section{Objetivos}

A través de este estudio se pretende poner en valor el concepto, los pilares y el proceso de construcción de una escuela democrática, categorías sobre cuyos cimientos se asienta el carácter democrático de la institución escolar: la exigencia de la participación y la mejora de la convivencia, a través de la voz de especialistas de la educación de Salta (Argentina) y Valencia (España), comparando las acepciones más destacadas entre ambos países.

A raíz de este objetivo principal se configuran dos objetivos específicos: realizar un análisis de contenido deductivo con la información recabada en los grupos focales y las entrevistas; así como comparar los resultados, observando las diferencias detectadas entre Salta (Argentina) y Valencia (España) y entre ambos grupos de participantes.

\section{Metodología}

\section{Participantes}

En la investigación han participado un total de 680 personas (Tabla 1), 350 proceden de Valencia (España) -250 alumnado de los grados de Pedagogía (Ped) y Educación Social (E. Soc) y 100 profesores/as de educación primaria (E. prim), secundaria (E. Sec), educación de adultos (E. Ad) y educación superior (E. Sup)- y 330 de Salta (Argentina) -210 alumnado de las titulaciones de Ciencias de la Educación (CC.EE) y de Profesorado en Letras (Prof. Let) y 120 profesores y profesoras de los mismos ámbitos especificados anteriormente. Esta muestra fue seleccionada de una manera no probabilística, ofreciendo la oportunidad de participar a aquellas personas que cumplían las características que la investigación demanda, lo que implica contar también con algunos profesionales dedicados a la gestión de las instituciones escolares.

Tabla 1- Características de la muestra de Salta y Valencia.

\begin{tabular}{|c|c|c|c|c|c|c|c|c|c|c|}
\hline & \multicolumn{4}{|c|}{ Profesorado } & & \multicolumn{4}{|c|}{ Alumnado } & \multirow[b]{2}{*}{ Total } \\
\hline & E. Prim & E. Sec & E. Ad & E. Sup & Total & CC. EE & Prof. Let & Ped & E. Soc & \\
\hline Salta, Argentina & 69 & 25 & 6 & 50 & 150 & 148 & 62 & & & 210 \\
\hline Valencia, España & 38 & 27 & 10 & 25 & 100 & & & 159 & 91 & 250 \\
\hline
\end{tabular}

Fuente: Elaboración propia.

\section{Instrumentos}

De acuerdo con el contexto y las posibilidades tanto en Valencia (España) como en Salta (Argentina) se ha optado por tres técnicas de recogida de información, grupos focales, entrevistas y encuestas, con la finalidad de llegar a un número elevado de participantes, visitando centros educativos situados en zonas de difícil acceso. Cabe destacar que en las tres técnicas se emplearon las mismas preguntas y ámbitos temáticos (Tabla 2). 
Tabla 2- Ámbitos y preguntas principales de los grupos focales y de las entrevistas realizadas

\begin{tabular}{c:c}
\hline Ámbito & Pregunta \\
\hline Concepto de educación democrática & ¿Qué entiende por educación democrática? \\
\hline Pilares de una escuela democrática & ¿Considera que la educación democrática debe ser la clave para trasformar la escuela de hoy? \\
\hline & ¿La participación y la convivencia son los pilares en la construcción de una escuela \\
& democrática? ¿Por qué? \\
\hline Construcción de una escuela democrática & ¿Q'ómo debe fomentarse la participación en la escuela? \\
\hline & ¿Qué estrategias deben estar presentes en el proceso de construcción de una escuela \\
& ¿Cómo podríamos mejorar la convivencia en la escuela? \\
\hline & ¿Qómo se debe construir una escuela democrática? \\
\hline
\end{tabular}

Fuente: Elaboración propia.

Tabla 3- Distribución de la muestra por técnica de recogida de información, país y grupo de interés

\begin{tabular}{|c|c|c|c|c|}
\hline \multirow[t]{2}{*}{ Técnica de recogida de información } & \multicolumn{2}{|c|}{ Salta (Argentina) } & \multicolumn{2}{|c|}{ Valencia (España) } \\
\hline & Alumnado & Profesorado & Alumnado & Profesorado \\
\hline Grupo Focal (G.F) & $\begin{array}{c}6 \text { grupos } \\
\text { (87 estudiantes) }\end{array}$ & $\begin{array}{c}5 \text { grupos } \\
\text { (67 profesores) }\end{array}$ & $\begin{array}{c}7 \text { grupos } \\
\text { (95 alumnos) }\end{array}$ & $\begin{array}{c}5 \text { grupos } \\
\text { (71 profesores) }\end{array}$ \\
\hline Entrevista semi-estructurada & 27 & 33 & 31 & 26 \\
\hline Encuesta & 48 & 29 & 124 & 32 \\
\hline Total & 210 & 120 & 250 & 100 \\
\hline
\end{tabular}

Fuente: Elaboración propia.

\section{Grupo Focal}

El grupo focal se trata de una técnica de investigación dirigida a recabar información de una determinada temática, mediante la interacción y el debate grupal (KRUEGER; CASEY, 2000; MORGAN, 1998). Gracias a este método se puede establecer un clima de discusión que permite conocer los significados que los participantes otorgan al tema principal.

\section{Entrevista semiestructurada}

Por otra parte, la entrevista semiestructurada ayuda a recopilar información expresada de forma oral por las personas participantes en la investigación, quienes comparten con el/la investigador/a la temática de interés que se aborda en el presente estudio (FONTANA; FREY, 2005). En la realización de las entrevistas, el contacto entre los diferentes participantes crea un clima enriquecedor para las dos partes, consiguiendo establecer una relación cercana donde las figuras del investigador y del sujeto objeto de estudio se desdibujan hasta llegar a convertirse en conversaciones íntimas entre personas con intereses comunes. 


\section{Encuesta}

La encuesta es una técnica de recogida de información a la que recurren gran número de investigadores para poder llegar a un número más elevado de participantes, ya que facilita el acceso a la información (PÉREZ JUSTE, 2006), tanto de manera oral como de manera escrita, como es nuestro caso. En la presente investigación, debido al contexto, países participantes y al tiempo de la investigación, se han facilitado las preguntas anteriormente expuestas por escrito al alumnado y profesorado de ambos países, con la finalidad de lograr su participación en el proceso de recogida de información.

\section{Procedimiento}

Como se ha mencionado anteriormente, se realizaron tanto grupos focales (G.F) como entrevistas durante el curso 2014/2015, tanto en Valencia (España) como en Salta (Argentina). En ambos casos se atendió muy cuidadosamente a todas las personas participantes, teniendo en cuenta la importancia de generar tranquilidad y confianza. El tiempo fue un factor fundamental (40-45 minutos), ya que, dependiendo del caso, se profundizaba más o menos en determinados ámbitos temáticos.

\section{Análisis de datos}

El análisis de la información obtenida tanto de los grupos focales como de las entrevistas se ha llevado a cabo a través de un análisis de contenido inductivo, mediante el establecimiento de un sistema categorial previo, conformado por unidades que comparten significados afines en función de las intervenciones realizadas por los/as participantes en cada uno de los grupos (BARDÍN, 1986). Las categorías quedan establecidas de forma previa, coincidiendo con los ámbitos que han guiado los grupos focales y las entrevistas, al objeto de buscar las respuestas y poder analizar la información recabada a través de un hilo conductor. Es importante mencionar que, mediante el sistema categorial inductivo, se pretende guiar la investigación desde su inicio, con el objetivo de delimitar el ámbito de estudio relevante en el desarrollo del estudio.

Figura 1- Categorías de análisis

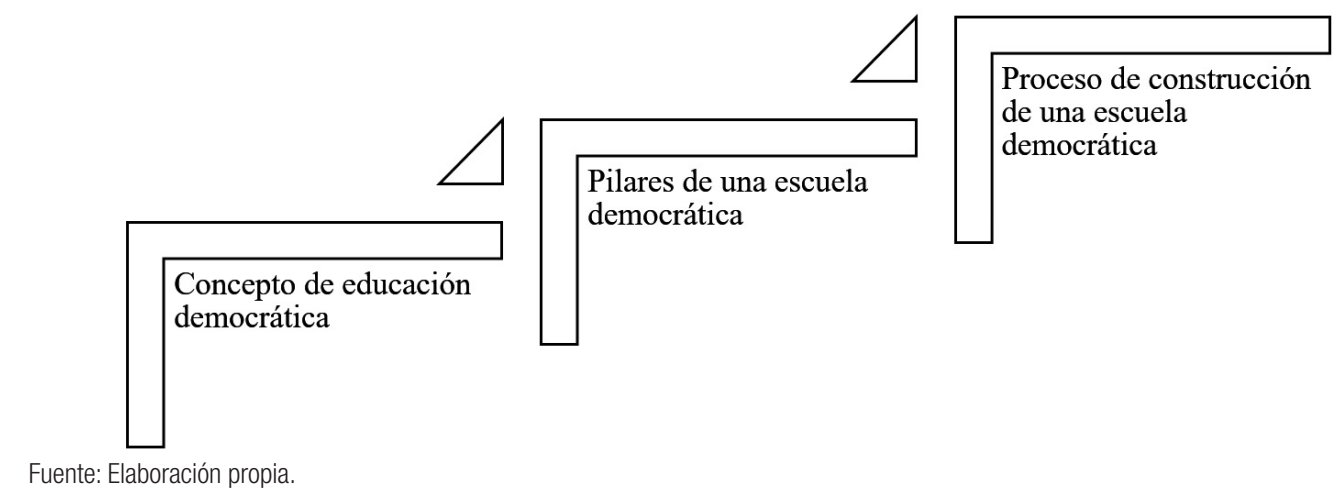


La información obtenida se ha procesado mediante el programa de análisis Atlas.ti ${ }^{3}$, al objeto de obtener el conteo de palabras y categorías afines mediante una metodología cualitativa (DENZIN; LINCOLN, 1994; MILES; HUBERMAN, 1994). A través de este programa pudimos extraer las palabras más repetidas en las transcripciones, además de construir los mapas conceptuales correspondientes que relacionan sus significados. Además, para finalizar el proceso y proponer una serie de conclusiones al respecto, se ha optado por aproximarnos a un análisis comparativo entre la información recogida en dos sentidos: comparación de resultados entre Valencia y Salta y entre el profesorado y el alumnado de ambos países.

\section{Resultados}

Ante las diferentes cuestiones que se han abordado, tanto en las entrevistas como en los grupos focales (GF), se ha procedido a analizar las respuestas de acuerdo con las categorías establecidas previamente en el proceso de análisis de contenido.

\section{a) Concepto de educación democrática.}

Las respuestas obtenidas por el alumnado de las titulaciones de Ciencias de la Educación y de Profesorado en Letras (Salta, Argentina) versan en torno a la necesidad de promover a través de la educación una sociedad más justa, igualitaria y comprometida.

La educación democrática es aquella que apuesta por la construcción de una Ciudadanía Emancipada. Esta ciudadanía debe promover y trabajar con los derechos políticos, civiles y sociales de todos los seres humanos. De manera que, integre a todos los sujetos que se encuentren en situación de pobreza y vulnerabilidad social, logrando una mayor equidad. (Entrevista. Estudiante de CC.EE, Salta, 2015).

Además, dejan claro que es el Estado y por ende el Gobierno quienes deben apostar por una educación democrática y es así como lo manifiestan.

Nuestro gobierno argentino tiene en sus manos promover instituciones donde se propicie la participación de todos sus integrantes. (...) [En las instituciones] todos sus miembros tienen que ser considerados y tratados de manera pareja e igual, eso es educar en democracia. Todos son escuchados y tenidos en cuenta en las distintas actividades que se realicen. Se pueden dar opiniones, y todas, digo, todas, deben ser respetadas y escuchadas. (GF. Estudiantes de Profesorado en Letras, Tartagal, 2015).

El alumnado de las titulaciones de Pedagogía y de Educación Social (Valencia, España), consideran que una educación democrática debe ser aquella que forme integralmente, dándole importancia al crecimiento personal y social con el propósito de aprender a vivir en una sociedad cambiante e intercultural.

\footnotetext{
3- Versión 6.02 bajo licencia de la UVEG (Universitat de València Estudi General).
} 
Una educación democrática entiendo que es una forma de educar en la que se cuente con todos y con todas, sin dejar a nadie fuera. Además, debe luchar y esforzarse por formar a cada una de las personas de manera integral... Sin olvidar la música, la educación física, el arte, las habilidades sociales (...). (GF. Estudiantes de Educación Social, Valencia, 2015).

En algunas ocasiones, varios estudiantes han hecho referencia también a la educación democrática como una forma más de promover el pensamiento crítico, con el propósito de formar a ciudadanos y ciudadanas activas y participativas. "La que educa para que aprendamos a vivir en sociedad pero, además, la que te ayuda a pensar para formar parte del proceso de transformación de esta sociedad injusta e insolidaria" (GF. Estudiantes de Pedagogía, Valencia, 2015).

Si por el contrario analizamos las respuestas obtenidas por el profesorado de los diferentes niveles educativos de Salta (Argentina), observamos que también siguen la misma línea que el alumnado del mismo país, otorgando principalmente el poder al Estado Argentino, pero sin excusar al profesorado, considerado el principal ejecutor del proceso de democratización de la educación. "[Educación democrática] Es lo mejor sí, pero para ello nos deben dar los recursos necesarios. Nosotros necesitamos capacitaciones para que realmente sepamos cómo construir una escuela democrática” (GF. Profesorado de CC.EE).

La preocupación por el papel de la familia en la escuela también es otro aspecto que sin duda varios maestros y maestras han destacado.

La educación democrática es respeto y es también trabajo conjunto, y las familias y la escuela deberían estar más unidos sí. Abrir espacios, nuevos lugares de intercambio para que comunidad y escuela caminen de la mano. Se tienen que escuchar si y necesitamos dialogar para construir un proyecto a partir de ambas miradas y visiones. (Entrevista. Maestra de Infantil, Valencia, 2015).

Es importante destacar que la reflexión sobre el proceso de democratización resulta evidente. Se ha mencionado en numerosas ocasiones la necesidad de formar al alumnado integralmente, pero demandan formación para saber cómo afrontar la educación de hoy.

[Educación democrática] es aquella que respeta a cada una de las personas de forma integral, atendiendo a las dificultades y apoyando a todas las personas que constituyen el centro educativo. (...) Es una escuela donde todos deben participar construyendo una comunidad más fuerte, valorando la escuela como clave para el desarrollo social, personal y cultural. (...) Debemos buscar cómo construir una escuela democrática, necesitamos estrategias y capacitaciones para ello, porque yo no sé cómo hacerlos ni por donde empezar. El Estado debe ayudarnos, él tiene el poder, pero somos nosotros quienes estamos aquí, enseñándoles a ser mejores personas, nosotros lo hacemos pero él nos tiene que ayudar (...).” (Entrevista. Maestra Primaria, Valencia, 2015).

El profesorado de Valencia (España) también menciona la necesidad de recibir apoyo del gobierno local para crecer como profesionales, haciendo referencia a la formación continua del profesorado, así como al apoyo económico para el desarrollo de proyectos de innovación educativa, clave para democratizar la educación. 
[Educación democrática] Debe apostar por la puesta en marcha de proyectos que incluyan a toda la comunidad educativa, todos son parte activa de la educación. Escuchar todas las voces, tenerlas en cuenta, tomar decisiones en busca de un camino conjunto (...). Las administraciones deben ayudarnos, formarnos en aspectos que fomenten el cambio. Queda muchísimo camino por delante, pero parece que eso a ellos no les alarma, no saben cómo estamos, en las condiciones en las que está la escuela de hoy. Claro, no lo ven. (GF. Profesorado de diferentes niveles educativos, Valencia, 2015).

\section{b) Pilares de una escuela democrática.}

El alumnado salteño explica y profundiza en que además de la participación y de la convivencia, los pilares de una escuela democrática deben ser aquellos que fomenten la inclusión de toda la población que forma parte de la institución educativa. Exponen, en este sentido, valores como la igualdad, el respeto y la libertad, desde una perspectiva que busca la educación de las personas para la igualdad de oportunidades y para la formación de un pensamiento crítico, entendiendo que uno de los objetivos de la escuela debe ser la construcción de una ciudadanía responsable. La responsabilidad de fomentar ese proceso de democratización la otorgan a los maestros y a las maestras, sin olvidar la necesaria formación. "Los pilares de una escuela democrática son: inclusión, aceptación, respeto, igualdad, libertad de elección, libertad de expresión. (...) Debemos capacitar a nuestros profesores, ellos cambiaran la escuela de hoy, ellos son quienes tienen el saber para hacerlo" (Encuesta. Estudiante de Profesorado en Letras).

Las cuestiones sociales y culturales son también parte de la preocupación de varios estudiantes, quienes constatan la necesidad de trabajar por conseguir una sociedad justa y solidaria con todos los pueblos argentinos.

Los pilares de las escuelas democráticas deben ser aquellos que atiendan las cuestiones sociales, de todo el pueblo, sin distinción de clases, razas o etnias culturales. Una escuela democrática es aquella dispuesta a escuchar la voz de los niños, jóvenes y adolescentes; una escuela capaz de incluir propuestas y nuevos desafíos para la educación. Una escuela democrática también debe ser transformativa, para poder convertir a los jóvenes en sujetos emancipados. La escuela democrática es la escuela soñada pero no lejos de hacerse posible. (Encuesta. Estudiante de CC.EE, Salta, 2015).

En esta línea, el alumnado valenciano hace especial hincapié en enumerar estrategias que fomenten la participación de la comunidad educativa en general, así como aquellas que apuesten por una convivencia pacífica y libre de estereotipos. "Talleres para las familias, profesorado y alumnado, además de invitar a organizaciones y entidades que apuesten por un modelo de escuela diferente" (Entrevista. Estudiante de Educación Social, Valencia, 2015).

Son conscientes de la necesidad de formar al profesorado en dichas estrategias ya que expresan conocer el funcionamiento de los centros educativos en los que destacan la demanda de los profesionales en cursos para seguir innovando. "Hacen falta estrategias que ayuden al profesorado a mejorar la escuela: mediación, talleres, celebración de festividades, programas de innovación, metodologías cooperativas, creación de grupos heterogéneos, estrategias de comunidades de aprendizaje (...)” (Entrevista. Estudiante de Pedagogía, Valencia, 2015). 
Respecto al profesorado argentino, sigue la misma línea que el alumnado salteño, atribuyéndose la responsabilidad de construir una escuela más democrática, además de demandar formación al gobierno del país.

Tenemos que enseñarles a pensar, a participar y a proponer soluciones a los reclamos. Las familias pueden participar sí, pero también cuando se le pida, para que propongan cosas, tienen que aprender a participar con argumentos y así se van construyendo esta escuela. (...) Se fomenta que los niños participen en la escuela, turnos de conversación, de intercambio, y que aprendan a argumentar, es un contenido, aprender a razonar, que sepan defender su posición crítica y que aprendan a respetar la opinión del otro. Hay que resignificar la función del docente y la función de la escuela. Al existir tantas problemáticas sociales se desvirtúa la dinámica de la institución. Estamos creando mentes y queremos que sean mentes brillantes. (Entrevista. Directora de Escuela, Tartagal, 2015).

En Valencia también se remarca la necesidad de formación, pero en estrategias determinadas para la promoción de la participación y de la convivencia, con el objetivo de dar respuesta a las necesidades que la educación de hoy manifiesta.

[El alumnado] Necesita saber afrontar los conflictos que surgen cada día en el centro, las familias deben aprender a reaccionar de forma diferente y a participar en la escuela sin imponer sus ideas y su forma de educar. Pero [el profesorado], debemos atender a las necesidades que demanda la comunidad educativa, formarnos para saber cómo promover de manera práctica la participación y la mejora de la convivencia. Programas como el de alumnos ayudantes, asamblea de aula y de centro, actividades cooperativas y colaborativas, inteligencias múltiples... Pero para todo ello, necesitamos formación y tiempo. (Entrevista. Profesor de Secundaria, Valencia, 2015).

\section{c) Construcción de una escuela democrática.}

Finalmente, respecto al proceso de construcción de una escuela democrática, el alumnado de ambas titulaciones de Salta reflexiona y profundiza sobre dicho concepto, quienes proponen una escuela que presentan como un modelo ideal a seguir.

[Escuela democrática] debe construirse de manera que no tenga restricciones de acceso por algún tipo de discriminación racial, religiosa (...). Una escuela que sea abierta a la comunidad, a los estudiantes, que le brinde un espacio para la comunicación y que tenga en cuenta sus reales necesidades. (Encuesta. Estudiante de Profesorado en Letras, Tartagal, 2015).

Hacen referencia en numerosas ocasiones a que el modelo de escuela democrática debe ser el que incluya a toda la población. Además, expresan la necesidad de que se otorgue mayor libertad al funcionamiento de la escuela, sin que el Estado ejerza tanto control sobre las mismas.

[Escuela democrática] Debería ser un espacio abierto que posibilite en los sujetos la reflexiónauto-reflexión sobre su realidad, sobre el entorno que lo rodea. Implicaría "dejar ser" al sujeto desde los diversos aspectos que lo atraviesan. Sería un ámbito de liberación de individuos 
oprimidos y buscaría la igualdad de oportunidades, un espíritu crítico y moral. Pensar en una escuela democrática me remite en pensar además en una escuela no parametrada por la normativa institucional impuesta y oficialista. Estaría abierta a todas las necesidades que la sociedad demanda. (GF. Estudiantes de CC.EE, Salta, 2015).

En Pedagogía y Educación Social se han centrado más en el proceso de construcción de una escuela real, reflexionando también en las características que debería tener una escuela diferente e ideal, pero asentando las bases para transformar la escuela de hoy.

[Escuela democrática] debe ser aquella que te forme para ser libre, sin que ello suponga acabar con la libertad de los demás, por supuesto. ¿Cómo la construiría? Partiendo de que los niños aprenden descubriendo, reflexionando, equivocándose (...). [Las familias] Deben estar también en la escuela, deben formar parte activa. (...) ¿Estrategias? Las propuestas por corrientes educativas que apostaban por un modelo de escuela participativo, activo y libre, sí, creo que sí. (GF. Estudiantes de Pedagogía, Valencia, 2015).

Consideran que tanto educadores/as como pedagogos/as deben ser promotores de cambio. Apuestan por transformar la escuela de hoy, más que por construir una escuela nueva, a través de estrategias que fomenten la creatividad y el aprendizaje significativo.

Yo no la construiría, sino más bien transformaría la de hoy (...). Introduciría más estrategias como la música como recurso de aprendizaje, o las artes plásticas, o me basaría en modelos de escuela como la de Freinet o la pedagogía Montessori, analizando la mejor manera de educar para formar a ciudadanos y ciudadanas que aprendan a mejorar la sociedad en la que hoy vivimos. (Entrevista. Entrevista Estudiante de Educación Social, Valencia, 2015).

El profesorado salteño de nuevo sigue la misma línea que el alumnado argentino, proponiendo un modelo de escuela diferente, pero demandando reiteradamente la formación necesaria para que algún día pueda cumplirse la que denominan la escuela soñada.

Es una escuela que valora al alumnado y a las familias, donde es fundamental que todos tengan acceso a la educación y puedan crecer democráticamente, pudiendo estudiar y ser quienes quieran ser. (...) Donde se escuche la voz de todas las personas y se creen espacios de diálogo y de debate. Donde se trabaje solidariamente, participativamente y conjuntamente hacia el mismo camino. Ahora sí, y yo te pregunto, ¿y eso cómo se hace? Yo no lo sé (...). (Entrevista. Maestro Primaria, Salta, 2015).

Observamos, una vez más, la preocupación por el interés de las familias por sus hijos e hijas. El profesorado pone de manifiesto la necesidad de que éstas entren a formar parte activa de la institución escolar.

[En la escuela] las familias deben participar más y deben interesarse más por cómo evolucionan sus hijitos, ya que actualmente no lo hacen muchos de ellos. (...) No es una escuela democrática aquella donde los maestros manden y los niños obedezcan. Es una escuela donde sí se valore a 
los niñitos y se les escuche, ellos también tienen cosas muy interesantes que decir. (Entrevista. Maestra Primaria, Salta, 2015).

El profesorado valenciano expresa firmemente que una escuela democrática se construye con la voluntad de todos y todas, pero remarcan especialmente la dificultad del tiempo como su principal enemigo.

Una escuela democrática debe impulsar la participación, la convivencia, la participación de las familias en la escuela (...). Apostar por llevar a cabo proyectos de innovación, comunicación con otras escuelas del barrio, asociaciones, entidades (...). Pero claro, es preciso saber cómo hacerlo, pero ¿cuándo? Con todo lo que la administración nos pide, las horas lectivas, la preparación de clases, la atención a familias (...). No nos queda tiempo, no hay más. El tiempo es nuestro principal enemigo. (GF. Profesorado Primaria, Valencia, 2015).

En última instancia, si observamos la Figura 2, podemos comprobar que agrupa los resultados obtenidos de los cuatro grupos de estudio en relación a las tres categorías de estudio, donde confluyen los puntos comunes, con la finalidad de exponer las respuestas y sintetizar la información recabada al respecto.

Figura 2- Síntesis de resultados por categoría de estudio

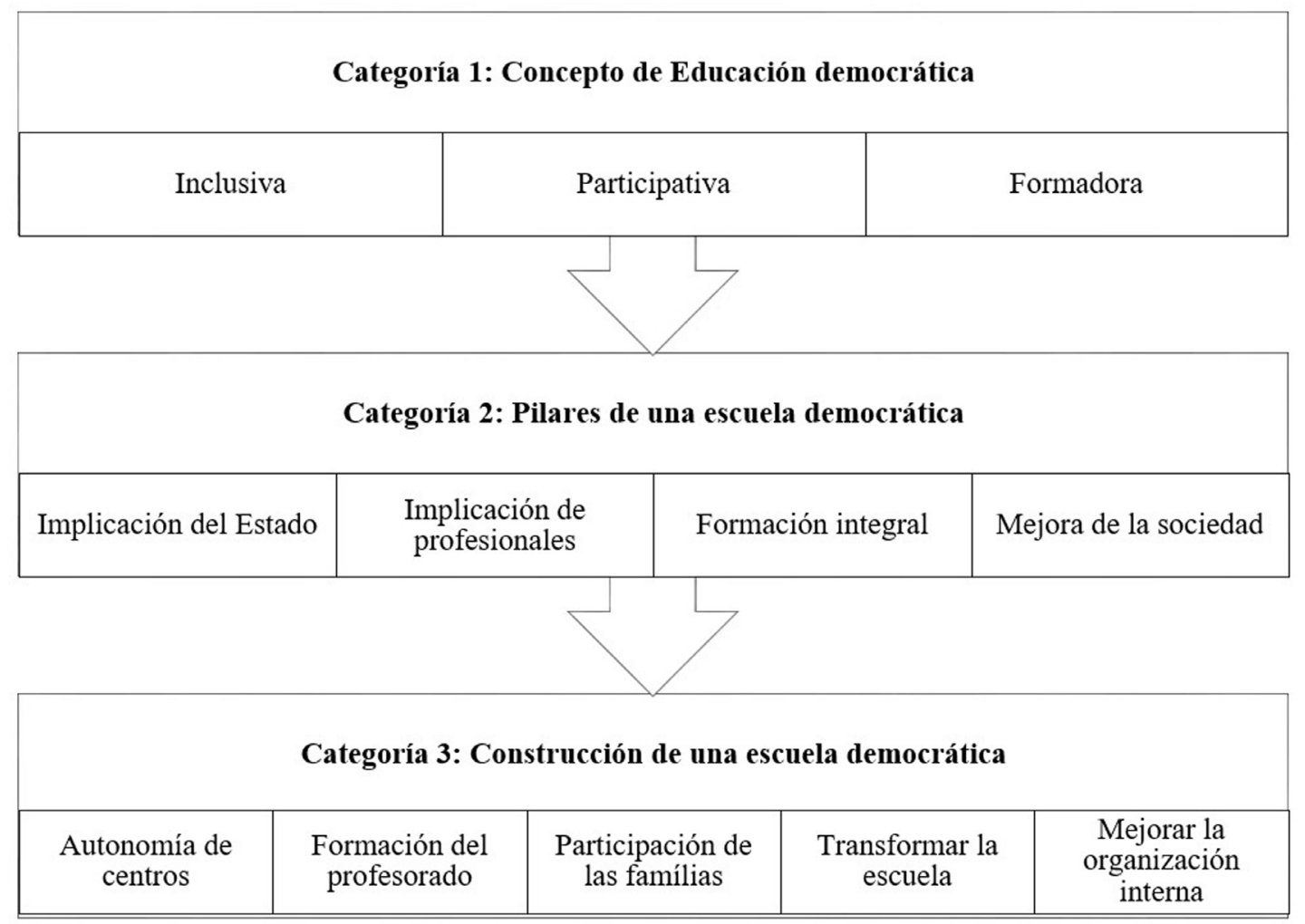

Fuente: Elaboración propia. 


\section{Conclusiones y discusión}

Resulta incuestionable el interés educativo por revitalizar la ciudadanía y trasmitir los valores en los que se apoya la democracia en las sociedades actuales. Una escuela democrática no sólo se fundamenta en la enseñanza de estos valores, sino que exige la gestión democrática de su funcionamiento. Participación, convivencia y formación para una ciudadanía responsable son los pilares sobre los que debe fomentarse la construcción de una escuela democrática. Aun cuando todos los análisis realizados reflejan escenarios que ponen en valor los aspectos mencionados, a la hora de concretar los componentes competenciales en los que deben desmenuzarse estos cimientos fundamentales, encontramos algunas diferencias de concepto que debemos subrayar, si queremos perfilar de forma precisa el significado de una escuela democrática.

Sin duda, un aspecto común señalado como un valor primordial para el adecuado desarrollo de una escuela democrática, quizás condición imprescindible, sea la participación de todos y cada uno de los sectores de la comunidad educativa. Las opiniones vertidas por los especialistas en educación y los propios estudiantes de Salta y de Valencia, señalan la práctica de la participación como una herramienta esencial para aprender a vivir democráticamente. Tal y como indica García Pérez (2009), la participación es concebida como un elemento fundamental para aprender a vivir en un mundo cada día más exigente, clave para aprender a afrontar problemas y para ser competente en la toma de decisiones. Por tanto, los centros educativos deben potenciar la mejora de la convivencia, promoviendo el desarrollo integral de toda la comunidad educativa (PAREJA; PEDROSA, 2013). Estamos en condiciones de afirmar que aprender a vivir en sociedad, a participar en la comunidad, no es una tarea fácil, pero sí debe ser un objetivo prioritario en el ámbito escolar: la participación se enseña, se aprende y debe experimentarse a nivel práctico.

Por el contrario, llama la atención las diferencias evidentes que se producen en cuanto al tratamiento diverso que los participantes de ambos países realizan respecto a las responsabilidades del profesorado y de la comunidad educativa en la construcción de una escuela democrática. Por una parte, tanto el alumnado como el profesorado de Salta (Argentina) se refiere al Estado como el principal impulsor del cambio educativo, asumiendo el profesorado la máxima responsabilidad para cumplir los deberes que el gobierno les trasmite. Sin duda, la crítica al mismo y la demanda de diferentes exigencias son evidentes en todas las entrevistas y grupos focales realizados. Por el contrario, en Valencia, tanto el alumnado como el profesorado son conscientes del apoyo que necesitan de las administraciones públicas, pero a la vez se sienten libres para construir con sus propios medios una escuela democrática, responsabilizando a la comunidad educativa y otorgando a la misma la necesidad de caminar hacia un mismo sentido. En ambos casos, la implicación de toda la comunidad educativa deberá ir acompañada del liderazgo institucional del profesorado y los gestores del centro escolar.

Sería oportuno profundizar en este análisis a la hora de concretar una adecuada formación del profesorado que le permita dotarle de herramientas para afrontar los retos que ellos mismos perciben de las experiencias vividas. Si bien parecen asumidos los planteamientos teóricos en cuanto a la importancia de capacitar al alumnado para el 
ejercicio de la libertad, en un contexto no exento de una acusada tendencia a la equidad, no parecen igualmente resueltas -quizás expresión del reconocimiento de un déficit formativola preparación en toda una serie de técnicas (mediación, aprendizaje colaborativo, educación emocional, etc.). De esta manera, se facilitaría la consecución de esos aspectos destacados como el fomento del diálogo, la comprensión del otro, la resolución pacífica de conflictos, etc. Acercar el discurso académico a la cultura práctica de la experiencia de este colectivo, resulta fundamental en una formación adecuada del profesorado.

Santos Guerra (2003), en esta línea, remarca la importancia de trabajar la empatía relacional entre los miembros de la comunidad educativa, incidiendo en que la construcción de una escuela democrática debe partir de un adecuado clima escolar, considerando la convivencia como un pilar fundamental ante cualquier proceso educativo; "[...] ser ciudadano hoy -escriben García Del Dujo y Mínguez (2011, p. 269)- es, ante todo, vivir y convivir con el entorno, donde resulta inevitable e imprescindible la presencia del otro y de lo otro". Quizás por ello, siguiendo estos planteamientos es por lo que el profesorado de ambos países ha considerado fundamental el trabajo conjunto de la comunidad educativa, incluyendo a las familias e incluso a las asociaciones ciudadanas; si bien es cierto que no ambos grupos les otorgan las mismas responsabilidades. Todos/as ellos/as son quienes deben apostar por transmitir a los niños y a las niñas de hoy la importancia de observar y reflexionar sobre el conjunto de la sociedad y de la tierra, y de forma más específica de las personas que la habitan, aprendiendo a formar parte de un mundo diverso y cambiante.

Cabe destacar, igualmente, que tanto la literatura científica, como la percepción de todas las audiencias implicadas, afirman que lo fundamental es buscar espacios y tiempos para poder ejercer la democracia, partiendo de la escuela como un escenario de interacción, de acción, de cooperación, que favorezca el aprendizaje significativo (DÍAZ AGUAD0, 2005). Resulta necesario crear un entorno que apueste por potenciar un clima social positivo, encargado de la búsqueda de las herramientas adecuadas para construir una realidad inclusiva, atendiendo a las necesidades integrales de la totalidad del alumnado (FELNER et al., 1995).

El grupo de estudiantes tanto de Salta como de Valencia, sin embargo, centra su atención en incrementar el protagonismo del alumnado, considerando que debe ser el profesorado quien lo facilite, con el objetivo de propiciar su empoderamiento en la toma de decisiones del centro. La unánime defensora de la importancia de la colaboración, sin duda, supone no sólo el reconocimiento de la necesidad de que el profesorado muestre una actitud de proximidad hacia el alumnado, sino de que todo el Plan Educativo de Centro (PEC) gire alrededor del estudiante situando a éste en el corazón de todos los procesos académicos y cotidianos de la vida de la institución docente.

Por tanto, después del análisis realizado, se puede concluir que una escuela democrática es una comunidad educativa donde todas las personas que la conforman buscan espacios y tiempos para el diálogo, la interacción, estableciendo compromisos comunes de colaboración; donde se respira alegría y creatividad, con respeto a las libertades individuales, apostando por la igualdad, donde debe olvidarse el yo en favor del nosotros, de la comunidad. Alimentar la democracia, impulsar la participación y fomentar la convivencia, en definitiva, debe ser el triple desafío de nuestra educación en 
el futuro inmediato, si queremos poner en valor su idoneidad para construir una sociedad vertebrada y comprometida.

\section{Referencias}

APPLE, Michael; BEANE, Jeames. Escuelas democráticas. Madrid: Morata, 1997.

BARDÍN, Laurence. El análisis de contenido. Madrid: Akal, 1986.

BOLÍVAR, Antonio. Ciudadanía y competencias básicas. Sevilla: Fundación Ecoem, 2008.

CANTARERO, Joan; MARTÍNEZ BONAFÉ, Jaume; MARTÍNEZ BONAFÉ, Àngels. Viure la democràcia a l'escola. Barcelona: Graó, 1999.

DENZIN, Norman; LINCOLN, Yvonna. Handbook of qualitative research. New York: Sage, 1994.

DÍAZ AGUAD0, María. La violencia entre iguales en la adolescencia y su prevención desde la escuela. Psicothema, Oviedo, v. 17, n. 4, p. 549-588, 2005.

FELNER, Robert et al. Socioeconomic disadvantage, proximal environmental experiences and socioemotional and academic adjustment in early adolescence: investigation of a mediated effects model. Child Development, Medford, v. 66, p. 774-792, 1995.

FERNÁNDEZ, Isabel. Los programas de ayuda para la mejora de la convivencia en instituciones educativas. Bordón, Madrid, v. 60, n. 4, p. 137-150, 2008.

FLECHA, Ramón. Los sueños son posibles, mejorar la realidad sin sueños es imposible. Escuela, Sevilla, v. 3.718, n. 1.074, p. 26-27, 2006.

FONTANA, Andrea; FREY, James. The interview, from neutral stance to political involvement. In: DENZIN, Norman; LINCOLN, Yvonna (Comp.). The Sage handbook of qualitative research. London: Sage, 2005. p. 695-727.

GARCÍA DEL DUJO, Ángel; MÍNGUEZ, Ramón. Los límites de la educación en valores cívicos: cuestiones y propuestas pedagógicas. Educación XXI, Madrid, v. 14, n. 2, p. 263-285, 2011.

GARCÍA PÉREZ, Francisco. Educar para la participación ciudadana: un reto para la escuela de siglo XXI. Investigación en la Escuela, Sevilla, v. 68, p. 5-10, 2009.

GARCÍA RAGA, Laura; LÓPEZ MARTÍN, Ramón. La convivencia escolar y la construcción de la ciudadanía: balance retrospectivo y desafíos de futuro. Bordón, Madrid, v. 66, n. 2, p. 93-106, 2014.

GARCÍA RAGA, Laura; MARTíNEZ USARRALDE, María; SAHUQUILLO, Piedad. Hacia una cultura de convivencia: la mediación como herramienta socioeducativa. Cultura y Educación, London, v. 24, n. 2, p. 207-217, 2012. 
GIMENO SACRISTÁN, José. ¿Qué es una escuela para la democracia? Cuadernos de Pedagogía, Barcelona, n. 275, p. 19-26, 1998.

GODÁS, Agustín; SANTOS REGO, Miguel; LORENZO, Mar. Convivir en los centros educativos. ¿Cómo lo ven alumnos, padres y profesores? Bordón, Madrid, v. 60, n. 1, p. 41-58, 2008.

GONZÁLEZ BAREA, Eva M. Convivencia intercultural en centros educativos multiculturales. ¿Hacia un reconocimiento de la diversidad cultural? Educación XXI, Madrid, v. 26, p. 225-240, 2008.

JARES, Xesús. Pedagogía de la convivencia. Barcelona: Graó, 2006.

KRUEGER, Richard; CASEY, Mary. Focus groups: a practical guide for applied researchers. 3. ed. Thousand Oaks: Sage, 2000.

LÓPEZ MARTÍN, Ramón. Apostar por la educación: construir ciudadanía: el desafío de las sociedades del s. XXI. In: MONTANÉ, Alejandra; SÁNCHEZ VALVERDE, Carlos (Coord.). Derechos humanos y educación social. Valencia: Germanía, 2013. p. 153-165.

MAYOR ZARAGOZA, Federico. Educación para la paz. Educación XXI, Madrid, v. 6, p. 17-24, 2003.

MILES, Mattheu B.; HUBERMAN, A. Michael. Qualitative data analysis. London: Sage, 1994.

MORGAN, David L. The focus groups guide book. Newbury Park: Sage, 1998.

PAREJA, José; PEDROSA, Beatriz. Mejora de la convivencia a través de la investigación-acción participativa. Profesorado, Granada, v. 3, n. 6, p. 467-491, 2013.

PÉREZ-JUSTE, Ramón. Evaluación de programas educativos. Madrid: La Muralla, 2006.

REKALDE, Itziar; VIZCARRA, María; MACAZAGA, Ana. La observación como estrategia de investigación para construir contextos de aprendizaje y fomentar procesos participativos. Educación XXI, Madrid, v. 17, n. 1, p. 201-220, 2014.

SACRISTÁN, David; MURGA, María. Educación democrática y cultura participativa: desarrollo del espíritu democrático a través de la educación. Madrid: Universidad Nacional de Educación a Distancia, 1994.

SANTOS GUERRA, Miguel (Coord.) Aprender a convivir en la escuela. Madrid: Akal, 2003.

SILVA, Aida María; TAVARES, Celma. A cidadania ativa e sua relação com a educação em direitos humanos. Revista Brasileira de Política e Administração da Educação, Goiânia, v. 27, p. 13-24, 2011.

TIRAD0, Ramón; CONDE, Sara. Análisis estructural de la gestión de la convivencia escolar en centros de buenas prácticas de Andalucía (España). Educación XXI, Madrid, v. 18, n. 2, p. 1-23, 2015.

TONUCCI, Francesco. ¿Se puede enseñar la participación? Investigación en la Escuela, Sevilla, v. 68, p. 11-24, 2009. 
TORRECILLA, Eva; OLMOS, Susana; RODRÍGUEZ, María. Efectos de la metodología didáctica sobre el aprendizaje de competencias para la gestión de conflictos en educación secundaria. Educación XXI, Madrid, v. 18, n. 2, p. 1-27, 2015.

TOURIÑÁN, José. Familia, escuela y sociedad civil. Agentes de educación intercultural. Revista de Investigación en Educación, Vigo, v. 7, p. 7-36, 2010.

VIGUER, Paz; SOLĖ, Núria. Escuela e iguales como contextos de socialización en valores y convivencia: una investigación participativa a través de un debate familiar. Cultura y Educación, London, v. 24, n. 4, p. 475-487, 2012.

Recibido en: 04.12 .2017

Revisiones en: 13.06.2018 Aprobado en: 07.08.2018

Roser Grau Vidal es doctora en Educación, diplomada en Magisterio y licenciada en Pedagogía. Profesora e investigadora en el Departamento de Educación Comparada e Historia de la Educación de la Facultad de Filosofía y Ciencias de la Educación de la Universitat de València (España). Sus líneas de investigación prioritarias son la educación democrática y la convivencia escolar, especialmente en contextos de vulnerabilidad social.

Laura García Raga es doctora y licenciada en Pedagogía. Profesora e investigadora en el Departamento de Teoría de la Educación de la Facultad de Filosofía y Ciencias de la Educación de la Universitat de València (España). Sus líneas de investigación prioritarias son la convivencia y mediación escolar.

Ramón López Martín es catedrático de Teoría e Historia de la Educación en el Departamento de Educación Comparada e Historia de la Educación de la Facultad de Filosofía y Ciencias de la Educación de la Universitat de València (España). Sus líneas de investigación fundamentales se centran en la perspectiva histórica de la escuela y la dimensión social de la política educativa. 